Commun. Fac. Sci. Univ. Ank. Ser. A1 Math. Stat.

Volume 68, Number 2, Pages 2079-2093(2019)

DOI: $10.31801 /$ cfsuasmas. 586095

ISSN 1303-5991 E-ISSN 2618-6470

http://communications.science.ankara.edu.tr/index.php?series=A1

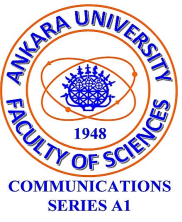

\title{
POSITION VECTORS OF CURVES WITH RECPECT TO DARBOUX FRAME IN THE GALILEAN SPACE $G^{3}$
}

\author{
TEVFİK ŞAHİN AND BUKET CEYLAN DİRİŞEN
}

\begin{abstract}
In this paper, we investigate the position vector of a curve on the surface in the Galilean 3-space $G^{3}$. Firstly, the position vector of a curve with respect to the Darboux frame is determined. Secondly, we obtain the standard representation of the position vector of the curve with respect to Darboux frame in terms of the geodesic, normal curvature and geodesic torsion. As a result of this, we define the position vectors of geodesic, asymptotic and normal line along with some special curves with respect to Darboux frame. Finally, we elaborate on some examples and provide their graphs.
\end{abstract}

\section{INTRODUCTION}

The fundamental theorem of curves state that curves are determined by curvatures [1. Thus, curvature functions provide us with some special and important information about curves. For example, a circular helix is a geometric curve with curvature $\kappa \equiv$ const. $\neq 0$, torsion $\tau \equiv$ const. $\neq 0$ [2. Straight lines and circles are curves that $\kappa \equiv 0$ and $\kappa \equiv$ cons., $\tau \equiv 0$, respectively. Also, these curves are degenerate helices. Helices appear in many different branch of science such as engineering, biology, chemistry, CAD, etc.

In addition, curvature functions give us information about not only curves but also surfaces on which curves lie. The geodesic curvature $\kappa_{g}$, normal curvature $\kappa_{n}$ and geodesic torsion $\tau_{g}$ charactarize geodesic, asymptotic curve, and line of curvature, respectively. The curves emerges from the solution of some important physical problems. They are also important in the theory of curves and surfaces. For example, geodesics arise from the problem of finding 'shortest curves' joining two points of a surface $M$. It was first considered by Johann Bernoulli (1697). Obviously this is a typically problem of calculus of variations. Also, a curve $C$ on a surface $M$ is called a geodesic curve or geodesic if its geodesic curvature $\kappa_{g}$ vanishes identically [1]. In what follows, we state three different definition lines in planes.

Received by the editors: April 22, 2018; Accepted: May 30, 2019.

2010 Mathematics Subject Classification. 53A35; 53B30.

Key words and phrases. Position vector, Darboux frame, geodesic, Galilean 3-space. 
We want to emphasize that geodesics can be seen as extension of this idea to curves in surfaces.

A "line" on a surface can be seen as extension of the familiar properties of lines in the plane: For example, lines are (1) the curves of shortest length joining two points (Archimedes). (2) The curves of plane curvature identically zero (Huygens, Leibniz, Newton). (3) The curves whose tangent and its derivative are linearly dependent 2 .

As stated in [3, the problem of the determination of the position vector of a space curve with respect to the Frenet frame is still open in the Euclidean space. Generally, it is hard to solve this problem. However, it is solved for some special curves such as plane curves, helix and slant helix [4, 5, 6]. On the other hand, in the Galilean space $G^{3}$, the foregoing problem is solved for all curves [3].

The main aim of this study is to solve the above problem for all curves on a surface in $G^{3}$ with respect to the Darboux Frame. Firstly, we determine the position vector of a curve on a surface in $G^{3}$ in terms of geodesic, normal curvature and geodesic torsion with respect to the Darboux and standard frame. Secondly, we shall give position vectors of some special curves such as geodesic, asymptotic curve, line of curvature on a surface in $G^{3}$.

Also, we will relate foregoing curves with helix, Salkowski curve and anti-Salkowski curve (see (30) ). That is, we shall give special cases of these curves such as: geodesics that are circular helix, genaralized helix or Salkowski, etc. Furthermore, we provide graphs of some special curves.

Last but not least, we want to emphasize that the results of this study can be extended to families of surfaces that have common geodesic curve.

\section{Preliminaries}

As it is well known, Galilean geometry is associated with the Galilean principle of relativity. The Galilean space $G^{3}$ is one of the Cayley-Klein spaces equipped with the projective metric of signature $(0,0,+,+)[7$. The absolute figure of the Galilean space is the ordered triple $\{w, f, I\}$, where $w$ is an ideal (absolute) plane, $f$ is a line (absolute line) in $w$, and $I$ is a fixed eliptic involution of points of $f$.

In non-homogeneous coordinates the group of motion of $G^{3}$ (i.e. the group of isometries of $G^{3}$ ) has the form define :

$$
\begin{aligned}
& \bar{x}=a_{1}+x, \\
& \bar{y}=a_{2}+a_{3} x+y \cos \varphi+z \sin \varphi, \\
& \bar{z}=a_{4}+a_{5} x-y \sin \varphi+z \cos \varphi,
\end{aligned}
$$

where $a_{1}, a_{2}, a_{3}, a_{4}, a_{5}$, and $\varphi$ are real numbers $[8$. If the first component of a vector is not zero, then the vector is called as non-isotropic, otherwise it is called isotropic vector [8]. 
The scalar product of two vectors $\mathbf{v}=\left(v_{1}, v_{2}, v_{3}\right)$ and $\mathbf{w}=\left(w_{1}, w_{2}, w_{3}\right)$ in $G^{3}$ is defined by

$$
\mathbf{v} \cdot G \mathbf{w}= \begin{cases}v_{1} w_{1}, & \text { if } v_{1} \neq 0 \text { or } w_{1} \neq 0 \\ v_{2} w_{2}+v_{3} w_{3}, & \text { if } v_{1}=0 \text { and } w_{1}=0 .\end{cases}
$$

If $\mathbf{v} \cdot{ }_{G} \mathbf{w}=0$, then $\mathbf{v}$ and $\mathbf{w}$ are perpendicular. In particular, every isotropic vector is perpendicular to every non-isotropic vector. The norm of $\mathbf{v}$ is defined by

$$
\|\mathbf{v}\|_{G}=\sqrt{|\mathbf{v} \cdot G \mathbf{v}|}
$$

Let $I \subset \mathbb{R}$ and let $\alpha: I \rightarrow G^{3}$ be a curve parameterized by arc length (we abbreviate as p.b.a.l) with curvature $\kappa>0$ and torsion $\tau$. If $\alpha$ is a curve p.b.a.l. that is,

$$
\alpha(x)=(x, y(x), z(x)),
$$

then the Frenet frame fields are given by

$$
\begin{aligned}
T(x) & =\alpha^{\prime}(x), \\
N(x) & =\frac{\alpha^{\prime \prime}(x)}{\left\|\alpha^{\prime \prime}(x)\right\|_{G}} \\
B(x) & =T(x) \times_{G} B(x) \\
& =\frac{1}{\kappa(x)}\left(0,-z^{\prime \prime}(x), y^{\prime \prime}(x)\right),
\end{aligned}
$$

where $\kappa(x)$ and $\tau(x)$ are defined by

$$
\kappa(x)=\left\|\alpha^{\prime \prime}(x)\right\|_{G}, \quad \tau(x)=\frac{\operatorname{det}\left(\alpha^{\prime}(x), \alpha^{\prime \prime}(x), \alpha^{\prime \prime \prime}(x)\right)}{\kappa^{2}(x)} .
$$

Also, where $\times_{G}$ is the Galilean cross product defined by

$$
\mathbf{v} \times{ }_{G} \mathbf{w}=\left|\begin{array}{ccc}
0 & \mathbf{e}_{2} & \mathbf{e}_{3} \\
v_{1} & v_{2} & v_{3} \\
w_{1} & w_{2} & w_{3}
\end{array}\right|
$$

for $\mathbf{v}=\left(v_{1}, v_{2}, v_{3}\right)$ and $\mathbf{w}=\left(w_{1}, w_{2}, w_{3}\right)[9]$.

The vectors $T, N$ and $B$ are called the tangent, the principal normal and the binormal vector field, respectively [9]. Therefore, the Frenet-Serret formulae can be written as

$$
\left[\begin{array}{l}
T \\
N \\
B
\end{array}\right]^{\prime}=\left[\begin{array}{ccc}
0 & \kappa & 0 \\
0 & 0 & \tau \\
0 & -\tau & 0
\end{array}\right]\left[\begin{array}{l}
T \\
N \\
B
\end{array}\right]
$$

Frame fields constitute a very useful tool for studying curves and surfaces. However, the Frenet frame $T, N, B$ of $\alpha$ is not useful to describe the geometry of surface $M$. Since $N$ and $B$ in general will be neither tangent nor perpendicular to $\mathrm{M}$. Therefore, we require another frame of $\alpha$ for study the relation between the geometry of $\alpha$ and $M$. There is such a frame field that is called Darboux frame field of $\alpha$ with respect to $M$. The Darboux frame field consists of the triple of vector fields $T, Q, n$. The first and last vector fields of this frame $T$ and $n$ are a unit tangent 
vector field of $\alpha$ and unit normal vector field of $M$ at the point $\alpha(x)$ of $\alpha$. Let $Q=n \times{ }_{G} T$ be the tangential-normal.

Theorem 1. Let $\alpha: I \subset \mathbb{R} \rightarrow M \subset G^{3}$ be a unit-speed curve, and let $T, Q, n$ be the Darboux frame field of $\alpha$ with respect to $M$. Then

$$
\left[\begin{array}{l}
T \\
Q \\
n
\end{array}\right]^{\prime}=\left[\begin{array}{ccc}
0 & \kappa_{g} & \kappa_{n} \\
0 & 0 & \tau_{g} \\
0 & -\tau_{g} & 0
\end{array}\right]\left[\begin{array}{l}
T \\
Q \\
n
\end{array}\right] .
$$

where $\kappa_{g}$ and $\kappa_{n}$ give the tangential and normal component of the curvature vector, and these functions are called the geodesic and the normal curvature, respectively [10.

Proof. We have

$$
\begin{aligned}
T^{\prime} & =\left(T^{\prime} \cdot{ }_{G} Q\right) Q+\left(T^{\prime} \cdot{ }_{G} n\right) n \\
& =\left(\alpha^{\prime \prime} \cdot{ }_{G} Q\right) Q+\left(\alpha^{\prime \prime} \cdot{ }_{G} n\right) n \\
& =\kappa_{g} Q+\kappa_{n} n .
\end{aligned}
$$

The other formulae are proved in a similar fashion.

Also, the equation (8) implies the important relations

$$
\kappa^{2}(x)=\kappa_{g}^{2}(x)+\kappa_{n}^{2}(x), \quad \tau(x)=-\tau_{g}(x)+\frac{\kappa_{g}^{\prime}(x) \kappa_{n}(x)-\kappa_{g}(x) \kappa_{n}^{\prime}(x)}{\kappa_{g}^{2}(x)+\kappa_{n}^{2}(x)}
$$

where $\kappa^{2}(x)$ and $\tau(x)$ are the square curvature and the torsion of $\alpha$, respectively. We refer to [8, 9, 11, 12] for detailed treatment of Galilean and pseudo-Galilean geometry.

\section{Position vectors of a curve in Galilean space}

In this section, we will get an arbitrary curve on a surface in $G^{3}$. We will analyze position vector of the curve with respect to the Darboux and standard frame in $G^{3}$. 
Theorem 2. The position vector $\beta(x)$ of an arbitrary curve on a surface with respect to the Darboux frame in the Galilean space $G^{3}$ is given by:

$$
\begin{aligned}
\beta(x)=\left(x+c_{1}\right) \mathbf{T} & +\left\{-\frac{\left(x+c_{1}\right) \kappa_{n}(x)}{\tau_{g}(x)}+\left(c_{2}-\int f(x) \tau_{g}(x) \sin [t(x)] d x\right) \sin [t(x)]\right. \\
& \left.-\left(c_{3}+\int f(x) \tau_{g}(x) \cos [t(x)] d x\right) \cos [t(x)]\right\} \mathbf{Q} \\
& +\left\{\left(c_{2}-\int f(x) \tau_{g}(x) \sin [t(x)] d x\right) \cos [t(x)]\right. \\
& \left.+\left(c_{3}+\int f(x) \tau_{g}(x) \cos [t(x)] d x\right) \sin [t(x)]\right\} \mathbf{n}
\end{aligned}
$$

where $f(x)=\frac{\lambda_{1}(x) \kappa_{g}(x)}{\tau_{g}(x)}-\left(\frac{\lambda_{1}(x) \kappa_{n}(x)}{\tau_{g}(x)}\right)^{\prime} \frac{1}{\tau_{g}(x)}$ and $t(x)=\int \tau_{g}(x) d x$.

Proof. Let $\beta(x)$ be an arbitrary curve on a surface in the $G^{3}$, then, we may express its position vectors with respect to the Darboux frame as follows:

$$
\beta(x)=\lambda_{1}(x) \mathbf{T}+\lambda_{2}(x) \mathbf{Q}+\lambda_{3}(x) \mathbf{n}
$$

where $\lambda_{1}(x), \lambda_{2}(x)$ and $\lambda_{3}(x)$ are differentiable functions of $x \in I \subset \mathbb{R}$. By differentiating (11) and using (7), we get

$$
\begin{aligned}
\lambda_{1}^{\prime}(x)-1 & =0 \\
\lambda_{1}(x) \kappa_{g}(x)+\lambda_{2}^{\prime}(x)-\lambda_{3}(x) \tau_{g}(x) & =0 \\
\lambda_{1}(x) \kappa_{n}(x)+\lambda_{2}(x) \tau_{g}(x)+\lambda_{3}^{\prime}(x) & =0
\end{aligned} .
$$

The first equation of 12 leads to

$$
\lambda_{1}(x)=x+c_{1}
$$

where $c_{1}$ is an arbitrary real constant. To solve 12 for $\lambda_{i}$, we use the following change of variable $t=\int \tau_{g}(x) d x$ so that

$$
\begin{aligned}
& \lambda_{1}(t)=\left(\lambda_{1} \circ x\right)(t), \\
& \tau_{g}(t)=\left(\tau_{g} \circ x\right)(t), \\
& \lambda_{2}(t)=-\frac{\lambda_{1}(t) \kappa_{n}(t)}{\tau_{g}(t)}-\dot{\lambda_{3}}(t) .
\end{aligned}
$$

Here, $" \cdot "$ stands for derivative with respect to $t$.

Substituting (14) into 12 we get the following equation

$$
\ddot{\lambda_{3}}(t)+\lambda_{3}(t)=\frac{\lambda_{1}(t) \kappa_{g}(t)}{\tau_{g}(t)}-\left(\frac{\lambda_{1}(t) \kappa_{n}(t)}{\tau_{g}(t)}\right) .
$$


The general solution becomes

$$
\lambda_{3}(t)=\left[c_{2}-\int f(t) \sin t d t\right] \cos t+\left[c_{3}+\int f(t) \cos t d t\right] \sin t
$$

where $c_{2}, c_{3}$ are arbitrary real constants and $f(t)=\frac{\lambda_{1}(t) \kappa_{g}(t)}{\tau_{g}(t)}-\left(\frac{\lambda_{1}(t) \kappa_{n}(t)}{\tau_{g}(t)}\right) \cdot$. By differentiating (16) and plug the resulting equation into (14), we obtain

$$
\lambda_{2}(t)=-\frac{\lambda_{1}(t) \kappa_{n}(t)}{\tau_{g}(t)}+\left[c_{2}-\int f(t) \sin t d t\right] \sin t-\left[c_{3}+\int f(t) \cos t d t\right] \cos t .
$$

As a result the equations (16) and 17 becomes

$$
\begin{aligned}
\lambda_{2}(x)=-\frac{\left(x+c_{1}\right) \kappa_{n}(x)}{\tau_{g}(x)} & +\left(c_{2}-\int f(x) \tau_{g}(x) \sin [t(x)] d x\right) \sin [t(x)] \\
& -\left(c_{3}+\int f(x) \tau_{g}(x) \cos [t(x)] d x\right) \cos [t(x)] .
\end{aligned}
$$

and

$$
\begin{aligned}
\lambda_{3}(x) & =\left(c_{2}-\int f(x) \tau_{g}(x) \sin [t(x)] d x\right) \cos [t(x)] \\
& +\left(c_{3}+\int f(x) \tau_{g}(x) \cos [t(x)] d x\right) \sin [t(x)]
\end{aligned}
$$

where $f(x)=\frac{\lambda_{1}(x) \kappa_{g}(x)}{\tau_{g}(x)}-\left(\frac{\lambda_{1}(x) \kappa_{n}(x)}{\tau_{g}(x)}\right)^{\prime} \frac{1}{\tau_{g}(x)}$ and $t(x)=\int \tau_{g}(x) d x$.

Substituting equations (13), (18) and (19) to (11) we obtain $(10)$. This completes the proof.

Theorem 3. The position vector $\beta(x)$ of an arbitrary curve on a surface with respect to the standard frame in the Galilean space $G^{3}$ is computed from the natural representation form:

$$
\begin{array}{r}
\beta(x)=\left(x, \int\left[\int\left(\kappa_{g}(x) S_{\tau_{g}}-\kappa_{n}(x) \int \tau_{g}(x) S_{\tau_{g}} d x\right) d x\right] d x,\right. \\
\left.\int\left[\int\left(\kappa_{g}(x) C_{\tau_{g}}-\kappa_{n}(x) \int \tau_{g}(x) C_{\tau_{g}} d x\right) d x\right] d x\right)
\end{array}
$$

where $C_{\tau_{g}}=\cos \left[\int \tau_{g}(x) d x\right]$ and $S_{\tau_{g}}=\sin \left[\int \tau_{g}(x) d x\right]$.

Proof. If $\beta(x)$ is a curve on a surface in Galilean space $G^{3}$, then the Frenet equations (7) are hold. It is easy to see that the following differential equation is obtained by using (7).

$$
\left(\frac{1}{\tau_{g}(x)} \mathbf{Q}^{\prime}(\mathbf{x})\right)^{\prime}=-\tau_{g}(x) \mathbf{Q}(\mathbf{x})
$$


The above equation can be written in the form

$$
\frac{d^{2} \mathbf{Q}}{d t^{2}}+\mathbf{Q}=0
$$

where $t$ is the new variable that equals to $t=\int \tau_{g}(x) d x$.

Thus, we can write $\mathbf{Q}$ as follows:

$$
\mathbf{Q}=(0, \sin [\theta(t)], \cos [\theta(t)])
$$

If we substitute 22 into 21 , and solve componentwise, we get the following two equations

$$
\dot{\theta}(t)= \pm 1, \quad \ddot{\theta}(t)=0
$$

which lead to $\theta(t)= \pm t= \pm \int \tau_{g}(x) d x$. Without loss of generality, we can assume that $\theta(t)$ has a positive sign. Then we get

$$
\mathbf{Q}(x)=\left(0, \sin \left[\int \tau_{g}(x) d x\right], \cos \left[\int \tau_{g}(x) d x\right]\right) .
$$

From (7), we obtain

$$
\begin{aligned}
\mathbf{n}(x) & =-\int \tau_{g}(x) \mathbf{Q}(x) d x \\
& =-\int \tau_{g}(x)\left(0, \sin \left[\int \tau_{g}(x) d x\right], \cos \left[\int \tau_{g}(x) d x\right]\right) d x+\mathbf{c}
\end{aligned}
$$

where $\mathbf{c}$ is a constant vector. Since the first component of normal vector is zero, then we can take $\mathbf{c}=(0,0,0)$, and then

$$
\mathbf{n}(x)=\left(0, \cos \left(\int \tau_{g}(x) d x\right),-\sin \left(\int \tau_{g}(x) d x\right)\right)
$$

From (23) and (7), we have

$$
\begin{aligned}
\mathbf{T}^{\prime}(x) & =\kappa_{g}(x) \mathbf{Q}(x)+\kappa_{n}(x) \mathbf{n}(x) \\
& =\kappa_{g}(x)\left(0, \sin \left[\int \tau_{g}(x) d x\right], \cos \left[\int \tau_{g}(x) d x\right]\right) \\
& +\kappa_{n}(x)\left(0,-\int \tau_{g}(x) \sin \left[\int \tau_{g}(x) d x\right] d x,-\int \tau_{g}(x) \cos \left[\int \tau_{g}(x) d x\right] d x\right) \\
= & \left(0, \kappa_{g}(x) \sin \left[\int \tau_{g}(x) d x\right]-\kappa_{n}(x) \int \tau_{g}(x) \sin \left[\int \tau_{g}(x) d x\right] d x\right. \\
& \left.\quad, \kappa_{g}(x) \cos \left[\int \tau_{g}(x) d x\right]-\kappa_{n}(x) \int \tau_{g}(x) \cos \left[\int \tau_{g}(x) d x\right] d x\right) .
\end{aligned}
$$


If we let $C_{\tau_{g}}=\cos \left[\int \tau_{g}(x) d x\right]$ and $S_{\tau_{g}}=\sin \left[\int \tau_{g}(x) d x\right]$, then we have

$$
\mathbf{T}^{\prime}(x)=\left(0, \kappa_{g}(x) S_{\tau_{g}}-\kappa_{n}(x) \int \tau_{g}(x) S_{\tau_{g}} d x, \kappa_{g}(x) C_{\tau_{g}}-\kappa_{n}(x) \int \tau_{g}(x) C_{\tau_{g}} d x\right) .
$$

Taking the integral of 26 with respect to, we get

$$
\begin{aligned}
\mathbf{T}(x)=(0, & \int\left(\kappa_{g}(x) S_{\tau_{g}}-\kappa_{n}(x) \int \tau_{g}(x) S_{\tau_{g}} d x\right) d x \\
& \left.\int\left(\kappa_{g}(x) C_{\tau_{g}}-\kappa_{n}(x) \int \tau_{g}(x) C_{\tau_{g}} d x\right) d x\right)+\mathbf{d}
\end{aligned}
$$

where $\mathbf{d}$ is a constant vector. Since the first component of tangent vector is one, we can take $\mathbf{d}=(1,0,0)$, and then

$$
\begin{array}{r}
\mathbf{T}(x)=\left(1, \int\left(\kappa_{g}(x) S_{\tau_{g}}-\kappa_{n}(x) \int \tau_{g}(x) S_{\tau_{g}} d x\right) d x,\right. \\
\left.\int\left(\kappa_{g}(x) C_{\tau_{g}}-\kappa_{n}(x) \int \tau_{g}(x) C_{\tau_{g}} d x\right) d x\right) .
\end{array}
$$

Integrating (28) with respect to $x$, we have

$$
\begin{array}{r}
\beta(x)=\left(x, \int\left[\int\left(\kappa_{g}(x) S_{\tau_{g}}-\kappa_{n}(x) \int \tau_{g}(x) S_{\tau_{g}} d x\right) d x\right] d x\right. \\
\left.\int\left[\int\left(\kappa_{g}(x) C_{\tau_{g}}-\kappa_{n}(x) \int \tau_{g}(x) C_{\tau_{g}} d x\right) d x\right] d x\right)
\end{array}
$$

where $C_{\tau_{g}}=\cos \left[\int \tau_{g}(x) d x\right]$ and $S_{\tau_{g}}=\sin \left[\int \tau_{g}(x) d x\right]$ which leads to the equation (20) and the proof is complete.

\section{Applications}

We begin a study of important special curves lying on surfaces. For example, geodesic, asymtotic and curvature (or principal) line. Let $\beta$ be regular curve on the oriented surface in $G^{3}$ with the curvature $\kappa$, the torsion $\tau$, the geodesic curvature $\kappa_{g}$, the normal curvature $\kappa_{n}$ and the geodesic torsion $\tau_{g}$.

Definition 4. We can say that $\beta$ is

$$
\begin{aligned}
\text { geodesiccurve } & \Longleftrightarrow \kappa_{g} \equiv 0, \\
\text { asymptotic curve } & \Longleftrightarrow \kappa_{n} \equiv 0, \\
\text { line of curvature } & \Longleftrightarrow \tau_{g} \equiv 0 .
\end{aligned}
$$


Also, We can say that $\beta$ is called:

\begin{tabular}{ccc}
$\kappa, \tau$ & & $\beta$ \\
\hline$\kappa \equiv 0$ & $\Longrightarrow$ & a straight line. \\
$\tau \equiv 0$ & $\Longrightarrow$ & a plane curve. \\
$\kappa \equiv$ cons. $>0, \tau \equiv$ cons. $>0$ & $\Longrightarrow$ & a circular helix or $\boldsymbol{W}$-curve. \\
$\frac{\tau}{\kappa} \equiv$ cons. & $\Longrightarrow$ & a generalized helix. \\
$\kappa \equiv$ cons., $\tau \neq$ cons. & $\Longrightarrow$ & Salkowski curve $[12,13]$. \\
$\kappa \neq$ cons. $\tau \equiv$ cons. & $\Longrightarrow$ & anti-Salkowski curve [13].
\end{tabular}

4.1. The position vector of a family of geodesic line in the Galilean space $G^{3}$.

Theorem 5. The position vector $\beta_{g}(x)$ of a family of geodesic line in Galilean space $G^{3}$ is given by

$$
\begin{aligned}
\boldsymbol{\beta}_{\mathbf{g}}(x)= & \left(x,-\iint \kappa_{n}(x) \int \tau_{g}(x) S_{\tau_{g}} d x d x d x,\right. \\
& \left.-\iint \kappa_{n}(x) \int \tau_{g}(x) C_{\tau_{g}} d x d x d x\right) .
\end{aligned}
$$

Proof. By using $\kappa_{g}(x) \equiv 0$ in the equation 20, we obtain the above equation.

Corollary 6. The position vector of a geodesic that is a circular helix is defined by the equation

$\boldsymbol{\beta}_{\mathbf{g}_{\mathrm{ch}}}(\mathbf{x})=\left(x,-\frac{e}{c^{2}} \cos \left(c x+c_{1}\right)+e_{1} x^{2}+e_{2} x+e_{3}, \frac{e}{c^{2}} \sin \left(c x+c_{1}\right)+f_{1} x^{2}+f_{2} x+f_{3}\right)$

where $c, c_{1}, e, e_{1}, e_{2}, e_{3}, f_{1}, f_{2}$ and $f_{3}$ are constants.

Proof. By using the definition (4) and the equations (9) in (5), we get the above equation.

Corollary 7. The position vector of a geodesic that is a generalized helix is defined by the equation

$$
\begin{array}{r}
\boldsymbol{\beta}_{\mathbf{g}_{\mathbf{g} \mathbf{h}}}(\mathbf{x})=\left(x, \iint \kappa_{n}(x)\left[\cos \left(d \int \kappa_{n}(x) d x\right)+d_{1}\right] d x d x,\right. \\
\left.-\iint \kappa_{n}(x)\left[\sin \left(d \int \kappa_{n}(x) d x\right)+d_{2}\right] d x d x\right)
\end{array}
$$

where $d, d_{1}$ and $d_{2}$ are constants.

Proof. By using the definition (4) and the equations (9), we obtain $\tau_{g}(x)=d \kappa_{n}(x)$. By using this equation in (5), we get the above equation. 
Corollary 8. The position vector of a geodesic that is a Salkowski curve is defined by the equation

$$
\begin{array}{r}
\boldsymbol{\beta}_{\mathbf{g}_{\mathbf{s}}}(\mathbf{x})=\left(x, m \iint\left(\cos \left(\int \tau_{g}(x) d x\right)+m_{1}\right) d x d x,\right. \\
\left.-m \iint\left(\sin \left(\int \tau_{g}(x) d x\right)+m_{2}\right) d x d x\right)
\end{array}
$$

where $m, m_{1}$ and $m_{2}$ are constants.

Proof. By using the definition (4) and the equations (9), we obtain $\kappa_{n}(x) \equiv d($ const.) and $\tau_{g}(x) \neq$ const.. By using this equation in (5), we get the above equation.

Corollary 9. The position vector of a geodesic that is a anti-Salkowski curve is defined by the equation

$$
\begin{array}{r}
\boldsymbol{\beta}_{\mathbf{g}_{\mathbf{a s}}}(\mathbf{x})=\left(x, \iint\left(\kappa_{n}(x)\left[\cos \left(b x+b_{1}\right)+b_{2}\right]\right) d x d x\right. \\
\left.-\iint\left(\kappa_{n}(x)\left[\sin \left(b x+b_{1}\right)+b_{3}\right]\right) d x d x\right)
\end{array}
$$

where $b, b_{1}, b_{2}$ and $b_{3}$ are constants.

Proof. By using the definition (4) and the equations (9), we obtain $\tau_{g}(x) \equiv b$ (const.) and $\kappa_{n}(x) \neq$ const.. By using this equation in (5), we get the above equation.

\subsection{The position vector of a family of asymptotic line in the Galilean} space $G^{3}$.

Theorem 10. The position vector $\beta_{a}(x)$ of a family of asymptotic line in Galilean space $G^{3}$ is given by

$$
\begin{array}{r}
\boldsymbol{\beta}_{\mathbf{a}}(x)=\left(x, \iint \kappa_{g}(x) \sin \left(\int \tau_{g}(x) d x\right) d x d x,\right. \\
\left.\iint \kappa_{g}(x) \cos \left(\int \tau_{g}(x) d x\right) d x d x\right)
\end{array}
$$

Proof. By using $\kappa_{n}(x) \equiv 0$ in the equation $(20)$, we obtain the above equation.

Corollary 11. The position vector of a asymptotic that is a circular helix is defined by the equation

$$
\boldsymbol{\beta}_{\mathbf{a}_{\mathbf{c h}}}(\mathbf{x})=\left(x,-\frac{e}{c^{2}} \sin \left(c x+c_{1}\right)+c_{2} x+c_{3},-\frac{e}{c^{2}} \cos \left(c x+c_{1}\right)+c_{4} x+c_{5}\right)
$$

where $c, c_{1}, c_{2}, c_{3}, c_{4}, c_{5}$ and $e$ are constants. 
Proof. By using the definition (4) and the equations (9) in (32), we obtain $\kappa_{g}(x) \equiv$ $e, \tau_{g}(x) \equiv c$ where $e$ and $c$ are constants. By using this relation in 32 , we get the above equation.

Corollary 12. The position vector of a asymptotic that is a generalized helix is defined by the equation

$$
\begin{array}{r}
\boldsymbol{\beta}_{\mathbf{a g h}}(\mathbf{x})=\left(x,-\frac{1}{k} \int \cos \left(k \int \kappa_{g}(x) d x\right) d x+k_{1} x+k_{2},\right. \\
\left.\frac{1}{k} \int \sin \left(k \int \kappa_{g}(x) d x\right) d x+k_{3} x+k_{4}\right)
\end{array}
$$

where $k, k_{1}, k_{2}, k_{3}$ and $k_{4}$ are constants.

Proof. By using the definition (4) and the equations (9), we obtain $\tau_{g}(x)=k \kappa_{g}(x)$ where $k$ is a constant. By using this relation in (32), we get the above equation.

Corollary 13. The position vector of a asymptotic that is a Salkowski curve is defined by the equation

$$
\boldsymbol{\beta}_{\mathbf{a}_{\mathbf{s}}}(\mathbf{x})=\left(x, \iint\left(e \sin \left(\int \tau_{g}(x) d x\right)\right) d x d x, \iint\left(e \cos \left(\int \tau_{g}(x) d x\right)\right) d x d x\right)
$$

where e is a constant.

Proof. By using the definition (4) and the equations (9), we obtain $\kappa_{g}(x) \equiv e($ const.) and $\tau_{g}(x) \neq$ const.. By using this equation in $(32)$, we get the above equation.

Corollary 14. The position vector of a asymptotic that is a anti-Salkowski curve is defined by the equation

$$
\boldsymbol{\beta}_{\mathbf{a}_{\mathbf{a s}}}(\mathbf{x})=\left(x, \iint\left(\kappa_{g}(x) \sin \left(d x+d_{1}\right)\right) d x d x, \iint\left(\kappa_{g}(x) \cos \left(d x+d_{1}\right)\right) d x d x\right)
$$

where $d$ and $d_{1}$ are constants.

Proof. By using the definition (4) and the equations (9), we obtain $\tau_{g}(x) \equiv d($ const.) and $\kappa_{g}(x) \neq$ const.. By using this equation in $(32)$, we get the above equation.

4.3. The position vector of a family of line of curvature in the Galilean space $G^{3}$. 
Theorem 15. The position vector $\beta_{c}(x)$ of a family of line of curvature in Galilean space $G^{3}$ is given by

$$
\begin{array}{r}
\boldsymbol{\beta}_{\mathbf{p}}(x)=\left(x, \iint\left(c_{1} \kappa_{g}(x)-c_{2} \kappa_{n}(x)\right) d x d x,\right. \\
\left.\iint\left(c_{3} \kappa_{g}(x)-c_{4} \kappa_{n}(x)\right) d x d x\right)
\end{array}
$$

where $c_{1}, c_{2}, c_{3}$ and $c_{4}$ are constants.

Proof. By using $\tau_{g}(x) \equiv 0$ in the equation (7), we obtain the above equation.

Corollary 16. The position vector of a line of curvature is a circular helix if and only if the below system of differential equations is satisfied.

$$
\begin{aligned}
& \kappa_{g}(x) \kappa_{g}^{\prime}(x)+\kappa_{n}(x) \kappa_{n}^{\prime}(x)=0, \\
& \kappa_{n}(x) \kappa_{g}^{\prime \prime}(x)-\kappa_{g}(x) \kappa_{n}^{\prime \prime}(x)=0 .
\end{aligned}
$$

Proof. By using the definition (30) and the equations (9), we get the above equation.

\section{Special Case:}

If $\kappa_{g}(x)$ and $\kappa_{n}(x)$ are constant functions, then the equation (34) is satisfied. Therefore, the line of curvature with $\kappa_{g}(x) \equiv$ const. and $\kappa_{n}(x) \equiv$ const. is a circular helix, and its position vector is defined by

$$
\boldsymbol{\beta}_{\mathbf{p}_{\mathbf{c h}}}(x)=\left(x, a_{1} x^{2}+a_{2} x+a_{3}, b_{1} x^{2}+b_{2} x+b_{3}\right)
$$

where $a_{1}, a_{2}, a_{3}$ and $b_{1}, b_{2}, b_{3}$ are constants.

Corollary 17. The position vector of a line of curvature is a generalized helix if and only if the below differential equation is satisfied.

$$
\begin{array}{r}
\kappa_{g}^{\prime \prime} \kappa_{n}^{3}+\kappa_{g}^{\prime \prime} \kappa_{n} \kappa_{g}^{2}-\kappa_{n}^{2} \kappa_{g} \kappa_{n}^{\prime \prime}-3 \kappa_{n}^{2} \kappa_{g}^{\prime} \kappa_{n}^{\prime}-3 \kappa_{n} \kappa_{g} \kappa_{g}^{\prime 2}+3 \kappa_{n} \kappa_{g} \kappa_{n}^{2} \\
-\kappa_{g}^{3} \kappa_{n}^{\prime \prime}+3 \kappa_{g}^{2} \kappa_{g}^{\prime} \kappa_{n}^{\prime}=0
\end{array}
$$

Proof. By using the definition (30) and the equations (9), we get the above equation.

Corollary 18. The position vector of a line of curvature is a Salkowski curve if and only if the following equation is satisfied:

$$
\kappa_{g} \kappa_{g}^{\prime}+\kappa_{n} \kappa_{n}^{\prime}=0
$$

Proof. By using the definition (30) and the equations $(9)$, we get the above equation. 

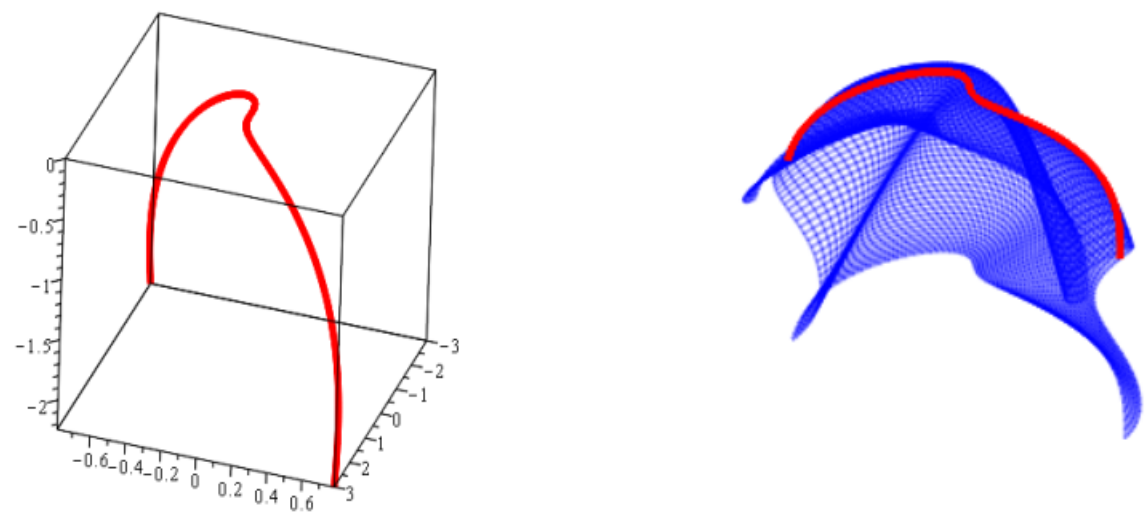

Figure 1. The geodesic curve and surface

Corollary 19. The position vector of a line of curvature is a anti-Salkowski curve if and only if the following equation is satisfied:

$$
\begin{aligned}
\kappa_{g}^{\prime \prime} \kappa_{g}^{2} \kappa_{n}+\kappa_{g}^{\prime \prime} \kappa_{n}^{3}-\kappa_{n}^{\prime \prime} \kappa_{g}^{3}-\kappa_{n}^{\prime \prime} \kappa_{g} \kappa_{n}^{2}-2 \kappa_{g}^{\prime 2} \kappa_{g} \kappa_{n}+2 \kappa_{g}^{\prime} \kappa_{n}^{\prime} \kappa_{g}^{2} \\
-2 \kappa_{g}^{\prime} \kappa_{n}^{\prime} \kappa_{n}^{2}+2 \kappa_{n}^{\prime 2} \kappa_{g} \kappa_{n}=0
\end{aligned}
$$

Proof. By using the definition (30) and the equations (9), we get the above equation.

We now consider an example for geodesic curve on surface along with their graphs.

Example 20. In (5), if we let $\kappa(x)=\sin x$ and $\tau(x) \equiv 1$, we obtain

$$
\alpha(x)=\left(x, \frac{x-\sin (x) \cos (x)}{4}, \frac{\sin (x)^{2}-x^{2}}{4}\right)
$$

A surface on which this curve lies can be taken as follows:

$$
\phi(u, v)=\left(u+v, \frac{u-\sin (u+v) \cos (u+v)}{4}, \frac{\sin (u+v)^{2}-u^{2}}{4}\right) .
$$

\section{Conclusions}

This study is obtained the position vectors of all curves on a surface in $G^{3}$ with respect to the Darboux Frame. Firstly, the position vector of a curve on a surface in $G^{3}$ in terms of geodesic, normal curvature and geodesic torsion with respect to the Darboux and standard frame is investigated. As result of these, position vectors 
of some special curves such as geodesic, asymptotic curve, line of curvature on a surface is obtained in $G^{3}$.

Consequently, relations of foregoing curves with helix, Salkowski curve and antiSalkowski curve are given(see (30) ). That is, special cases of these curves such as: geodesics that are circular helix, genaralized helix or Salkowski, etc is given. Furthermore, the graphs of some special curves is drawn .

In the light of these results, special smarandache curves with respect to Darboux frame in $G^{3}$ is studied in [15. Also, we want to emphasize that the results of this study can be extended to families of surfaces that have common geodesic, asymptotic curve and line of curvature.

Acknowledgement. This study was supported financially by the Research Centre of Amasya University (Project No: FMB-BAP16-0213). Also, the authors thank the referee for helpful suggestions.

\section{REFERENCES}

[1] Kreyszig, E., Differential Geometry, Dover Publications, Reprint, New York, 1991.

[2] McCleary, J., Geoemetry From a Differentiable Viewpoint, Cambridge University Press, 1994.

[3] Ali, A.T., Position vectors of curves in the Galilean space $G^{3}$, Matematički Vesnik, 64(3) (2012), 200-210.

[4] Ali, A.T., Position vectors of spacelike general helices in Minkowski 3-space, Nonlin. Anal. Theory Meth. Appl. 73(2010), 1118-1126.

[5] Ali, A.T., Position vectors of slant helices in Euclidean 3-space, Journal of the Egyptian Mathematical Society, 20(1) (2012), 1-6.

[6] Izumiya, S. and Takeuchi, N., New special curves and developable surfaces, Turk. J. Math. $28(2004), 531-537$.

[7] Molnar, E., The projective interpretation of the eight 3-dimensional homogeneous geometries, Beitr. Algebra Geom. 38(1997), 261-288

[8] Pavković, B.J. and Kamenarović, I., The equiform differential geometry of curves in the Galilean space, Glasnik Matematikči. 22(42) (1987), 449-457.

[9] Pavković, B.J., The general solution of the Frenet system of differential equations for curves in the Galilean space $G^{3}$, Rad HAZU Math. 450 (1990), 123-128.

[10] Şahin, T., Intrinsic equations for a generalized relaxed elastic line on an oriented surface in the Galilean space, Acta Math Sci. 33B(3) (2013) 701-711.

[11] Roschel, O., Die Geometrie des Galileischen Raumes, Habilitation Schrift, Leoben 1984.

[12] Yaglom, I. M. , A simple non-Euclidean geometry and its physical basis, Springer-Verlag, Nw York, 1979.

[13] Monterde, J., Salkowski curves revisted: A family of curves with constant curvature and non-constant torsion, Comput. Aided Geomet. Design. 26 (2009), 271-278.

[14] Salkowski, E., Zur transformation von raumkurven, Math. Ann. 66 (1909), 517- 557.

[15] Şahin, T. and Okur, M., Special Smarandache curves with respect to Darboux frame in Galilean 3-Space, Int. J. Adv. Appl. Math. and Mech. 5(3)(2018), 15-26 (ISSN: 2347-2529). 
Current address: Tevfik Şahin: Department of Mathematics, Amasya University, Amasya, Turkey.

E-mail address: tevfik.sahin@amasya.edu.tr, tevfiksah@gmail.com

ORCID Address: https://orcid.org/0000-0001-7598-5842

Current address: Buket Ceylan Dirişen: Department of Mathematics, Amasya University, Amasya, Turkey.

E-mail address: bceylandirisen@gmail.com

ORCID Address: https://orcid.org/0000-0002-8831-7373 